\title{
Intervención logopédica para la estimulación de las habilidades fonológicas en un caso con retraso madurativo
}

\author{
Pilar Noya Lameiro \\ Universidade da Coruña
}

\begin{abstract}
Resumen
En las últimas décadas el estudio de la conciencia fonológica fue un tema frecuente en la literatura científica, dado sus implicaciones en el desarrollo del lenguaje y de la lectura. El objetivo principal del presente trabajo fue valorar los efectos de un programa de intervención en conciencia fonológica en un caso de dificultades de aprendizaje asociadas a un retraso madurativo. Tras estimular las habilidades fonológicas deficitarias, los resultados muestran una mejora tanto en conciencia fonológica como en lectura, coincidiendo con estudios anteriores. Esta estimulación en personas con dificultades de aprendizaje puede ayudar a paliar sus dificultades, mejorando su rendimiento académico.
\end{abstract}

Palabras clave: conciencia fonológica, retraso madurativo, habilidades lectoras, dificultades de aprendizaje.

\section{Introducción}

En los últimos años el estudio de la conciencia fonológica ha adquirido una gran importancia, inducida sobre todo por la relación de reciprocidad establecida entre conciencia fonológica y lectura.

Proporcionar una definición clara y completa del término conciencia fonológica no es fácil, pues a lo largo del tiempo han ido variando las definiciones que se han proporcionado. En este estudio se siguió la definición aportada por Gómez-Betancur, Pineda y AguirreAcevedo (2005), que definen la conciencia fonológica como la capacidad para analizar y segmentar los componentes del habla (palabras, rimas, sílabas, sonidos, fonemas), y de efectuar operaciones complejas con ellos.

A su vez, son numerosas las investigaciones centradas en tratar de determinar la estructura de la conciencia fonológica. En este sentido y de acuerdo con Defior y Serrano (2011), podrían distinguirse los siguientes niveles: conciencia léxica, conciencia silábica, conciencia intrasilábica y conciencia fonémica.

La adquisición de los niveles de conciencia fonológica un orden decreciente, adquiriendo primero la conciencia léxica, luego silábica, después la intrasilábica y, por último, la conciencia fonémica (Herrera y Defior, 2005; Bandini, Santos y de Souza, 2013). Para un completo desarrollo de todos los niveles es necesario el aprendizaje de la lectoescritura (Alcock, Ngorosho, Deus y Jukes, 2010).

De Eslava y Cobos (2008) también apoyaron esta correlación entre ambas habilidades, indicando que las dificultades para desarrollar la conciencia fonológica durante los primeros años predecían futuros problemas de lectura.

Otra importante línea de investigación es aquella que intenta relacionar los déficits en conciencia fonológica con las dificultades del aprendizaje.

Siguiendo a Pérez-Vallejo (2010), las dificultades del aprendizaje pueden definirse como un retraso que no suele presentarse en todas las habilidades cognoscitivas, sino en alguna o algunas de ellas.

Las dificultades de aprendizaje pueden afectar pues a diferentes áreas (lectura, escritura, cálculo...). Por ello, las dificultades en conciencia fonológica son también frecuentes; así lo corrobora un estudio de Gómez, Duarte, Merchán, Aguirre y Pineda (2007).

Por lo tanto, si la conciencia fonológica suele estar afectada en niños con dificultades de aprendizaje y esta habilidad interfiere en el correcto desarrollo de la lectoescritura, su adquisición puede verse comprometida. Por este motivo, si se considera fundamental estimular el adecuado desarrollo de las habilidades fonológicas en niños con un desarrollo típico, tanto más conveniente será esta estimulación en niños con dificultades del aprendizaje. Una mayor destreza en este campo puede mejorar las dificultades que se suelen presentar con el aprendizaje de la lectoescritura $\mathrm{y}$, como consecuencia, mejorar el rendimiento académico de estos niños.

\section{Participantes}

\section{Método}

En este trabajo participó un niño de 7 años de edad (I. de aquí en adelante) que cursaba el $1^{\text {er }}$ curso de Educación Primaria. I. En el momento del estudio recibiendo ayuda logopédica desde hacía un año y ocho meses. Inicialmente se estableció una periodicidad de 1 sesión por semana de 30 minutos, pero posteriormente se aumentó a 2 sesiones semanales de la misma duración.

Por lo que respecta al ámbito familiar convive con sus padres, una hermana menor y con sus abuelos.

$\mathrm{Su}$ lengua materna es el gallego, aunque los datos que facilita la logopeda a la que acude I. informan de un bilingüismo mal integrado, confundiendo a menudo aspectos del gallego con el castellano, principalmente a nivel léxico y morfosintáctico. Estas dificultades se atribuyen a una escasa estimulación por parte de su ambiente más cercano, por ello se ofrecieron a la familia unas pautas dirigidas a mejorar dicha estimulación.

En función de los resultados de la evaluación inicial realizada por la logopeda del centro, se establece como diagnóstico probable un retraso madurativo, ya que se aprecian dificultades a nivel de varias áreas de desarrollo. 
Asociadas a dicho retraso el niño presenta dificultades de aprendizaje, especialmente de lectura y escritura.

Con el inicio del aprendizaje lector, las dificultades de aprendizaje se fueron haciendo más notables. Por este motivo se decidió iniciar un programa de trabajo destinado a mejorar la conciencia fonológica de I., pues hay estudios que señalan que la estimulación de esta área ayuda al correcto desarrollo de la lectoescritura.

\section{Instrumentos}

Durante la evaluación previa a la intervención se emplearon tres instrumentos. El primero fue el Cuestionario de Fluidez Neuropsicológico Infantil (CUMANIN) (Portellano, Mateos, Martínez-Arias, Granados y Tapia, 2000). Se trata de una escala que valora aspectos neuropsicológicos, ayudando a confirmar o descartar posibles disfunciones cerebrales, comparando los resultados obtenidos con los esperados para la edad del sujeto.

En segundo lugar se empleó la batería de Procesos Lectores Revisada (PROLEC-R) (Arribas, Cuetos, Rodríguez y Ruano, 2007). Esta batería está diseñada para evaluar la posible existencia de problemas lectores, permitiendo conocer aquellos procesos lectores que presentan dificultades.

Se completó esta fase de evaluación con la prueba de evaluación del Lenguaje Oral y Lenguaje Escrito (Loleva) (Fernández Amado, Mayor, Zubiauz, Tuñas, y Peralbo, 2006; Mayor, Zubiauz, Peralbo, Fernández Amado, Tuñas y Brenlla, 2011). El Loleva es una aplicación informática creada para ser utilizada por todos aquellos profesionales que se ocupan de la evaluación e intervención sobre el lenguaje oral y escrito. Está diseñada para analizar el nivel de conciencia fonológica y competencia lectora de los niños de Educación Infantil y primer ciclo de Educación Primaria.

Por lo que se refiere a la fase de intervención, durante todas las sesiones se emplearon varias de las actividades propuestas en el material de intervención Lenguaje Oral y Lenguaje Escrito (Lole) (Mayor y Zubiauz, 2011). Este material está destinado al desarrollo de la conciencia fonológica y a la preparación a la lectura. Se puede emplear con tres finalidades: adquisición de las habilidades de conciencia fonológica necesarias para la iniciación de la lectura, prevención de posibles dificultades de lectura en aquellos alumnos que puedan presentar dificultades de aprendizaje y reeducación de las dificultades de aprendizaje de la lectura, derivadas de un inadecuado desarrollo de la conciencia fonológica.

El Lole consta de un manual con 32 unidades (16 de rima y sílaba y 16 de fonemas), en las que se proponen diversas actividades con las que trabajar cada uno de los apartados de conciencia fonológica que se evalúan con la prueba Loleva. En este caso no se emplearon todas las unidades, sino que se seleccionaron aquellas dedicadas al reconocimiento y adición de sílaba y fonema, pues fueron las partes que decidieron reforzarse tras los resultados obtenidos después de la aplicación del Loleva en la fase de evaluación previa a la intervención.

\section{Procedimiento}

Este trabajo se realizó a lo largo de dos meses y medio. Durante ese tiempo, I. acudía a los servicios logopédicos dos veces a la semana, durante media hora cada día. Todo el estudio se realizó en un espacio logopédico adaptado.

Una vez establecido un clima cómodo y de confianza con el niño se comenzaron a aplicar las pruebas pertenecientes a la evaluación previa a la intervención. Esta fase se llevó a cabo a lo largo de siete sesiones, debido a la poca duración de cada sesión. En las dos primeras sesiones se aplicó el CUMANIN, para conocer el nivel de desarrollo general que tenía el sujeto. En segundo lugar se empleó el PROLEC-R, cuya aplicación también se completó en otras dos sesiones. La última de las tres pruebas empleadas fue el Loleva, aplicada a lo largo de tres sesiones.

Según los resultados obtenidos en la evaluación inicial se estableció como objetivo principal del trabajo la estimulación de las habilidades fonológicas deficitarias; reconocimiento y adición de sílaba y fonema, con el fin de mejorar las habilidades lectoras del niño. Los objetivos específicos establecidos fueron los siguientes:

- Estimular y/o mejorar la identificación de sílaba, posición inicial como final

- Estimular y/o mejorar la adición de sílaba inicial, en posición inicial y final

- Estimular y/o mejorar la identificación de fonema, en posición inicial y final

- Estimular y/o mejorar la adición de fonema, en posición inicial y final

Para lograr los objetivos propuestos se planteó una intervención dividida en 3 etapas a lo largo de 8 sesiones:

a) Etapa 1: Estimulación de los objetivos silábicos (sesiones 1, 2 y 3 ).

b) Etapa 2: Trabajo de los objetivos fonémicos. (sesiones 5, 6 y 7).

c) Etapa 3: Repaso de los contenidos aprendidos. (sesiones 4, y 8).

Las actividades que se plantearon en cada una de las etapas fueron tomadas del material LOLE. Todas las tareas fueron presentadas siguiendo una complejidad creciente para favorecer el aprendizaje de los contenidos.

Además de trabajarse los objetivos propuestos, se intentó reforzar la capacidad de atención, pues en algunas sesiones se mostraba dispersa.

Por último, se volvieron a evaluar los conocimientos lectores y fonológicos del niño con las pruebas PROLEC-R y Loleva, respectivamente. En esta última fase se emplearon un total de 5 sesiones.

\section{Resultados}

En este apartado se exponen los resultados obtenidos tanto en la evaluación previa a la intervención como en la realizada al finalizar la misma. En las variables que fueron objeto de estudio se compara el nivel de rendimiento en las pruebas aplicadas antes y después de la intervención, para comprobar si hubo o no una evolución positiva después de llevar a cabo el entrenamiento en habilidades fonológicas. 
La primera prueba empleada en la evaluación fue el Cuestionario de Fluidez Neuropsicológico Infantil (CUMANIN) (Portellano, Mateos, Martínez-Arias, Granados y Tapia, 2000). Los resultados muestran que el niño obtuvo tan sólo un percentil 13 en cuanto a su desarrollo verbal y un percentil de 34 a nivel no verbal. $\mathrm{Su}$ nivel de desarrollo general se corresponde con un percentil de 47, coincidiendo con un cociente de desarrollo inferior a la media. Estos resultados avalan el posible diagnóstico de retraso madurativo, pues se aprecian bajos niveles de desarrollo en todas las áreas. En la figura 1 se muestran las puntuaciones percentiles obtenidas en cada una de las subpruebas que componen este cuestionario.

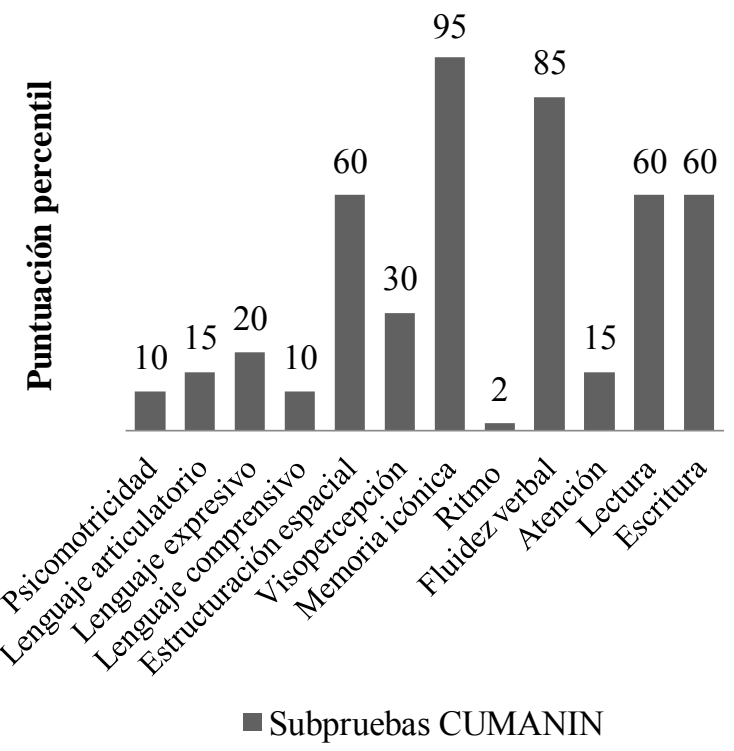

Figura 1. Puntuaciones percentiles obtenidas en las subpruebas del CUMANIN.

Como ya se comentó los resultados obtenidos por I. en el CUMANIN son bajos. A pesar de ello se aprecian puntos fuertes en los apartados de memoria icónica y fluidez verbal, donde obtuvo puntuaciones muy elevadas. En las pruebas de lectura, escritura y estructuración espacial también obtuvo unas puntuaciones aceptables. En el resto de variables analizadas obtuvo un percentil bajo o muy bajo, siendo en la de ritmo donde su rendimiento fue menor, ya que tan sólo obtuvo un percentil 2, sobre todo teniendo en cuenta que esta prueba está diseñada para niños más pequeños que I.

Respecto a la batería de Procesos Lectores Revisada (PROLEC-R) (Arribas, Cuetos, Rodríguez y Ruano, 2007), indicar que recoge datos acerca de las habilidades lectoras a través de tres escalas o índices: índices principales, índices de precisión e índices de velocidad.

En la escala índices principales se tienen en cuenta tanto los aciertos como el tiempo que invierte el niño en la realización de la tarea. Los resultados obtenidos por I. en este apartado se exponen en la tabla 1.
Tabla 1.

Resultados en índices principales del PROLEC $-R$ en las dos fases de evaluación

\begin{tabular}{ccc}
\hline Nombre de la subprueba & Evaluación Pre & Evaluación Post \\
\hline Nombre de Letras & Normal & Dificultad leve \\
Igual o Diferente & Normal & Normal \\
Lectura de Palabras & Dificultad leve & Normal \\
Lectura de & Normal & Normal \\
Pseudopalabras & & \\
Estructuras Gramaticales & Normal & Normal \\
Signos de Puntuación & Normal & Normal \\
Comprensión de & Normal & Normal \\
Oraciones & & \\
Comprensión de Textos & Dificultad leve & Normal \\
Comprensión Oral & Dificultad & Normal \\
& severa & \\
\hline
\end{tabular}

Al finalizar la intervención centrada en tareas de conciencia fonológica, se superan todas las dificultades que se presentaban en la evaluación inicial. En el apartado de nombre de letras se detecta una dificultad leve en la evaluación post-intervención, la cual no presentaba en la evaluación inicial.

Por lo que respecta a los índices de precisión, los resultados obtenidos se muestran en la tabla 2.

Tabla 2

Resultados en índices de precisión del PROLEC-R en las dos fases de evaluación

\begin{tabular}{ccc}
\hline Nombre de la prueba & Evaluación Pre & Evaluación Post \\
\hline Nombre de letras & Duda & Duda \\
Igual o diferente & Normal & Normal \\
Lectura de palabras & Dificultad leve & Normal \\
Lectura de & Normal & Normal \\
Pseudopalabras & & \\
Signos de puntuación & Duda & Normal \\
\hline
\end{tabular}

Se aprecian mejoras después de la intervención en todas las categorías que se encontraban en un nivel bajo en la evaluación inicial, excepto en el nombre de letras. En esta tarea había en los dos momentos de evaluación dudas sobre el nivel de rendimiento de I.

Finalmente, los resultados de índices de velocidad se muestran en la tabla 3.

Tabla 3

Resultados en índices de velocidad del PROLEC-R en las dos fases de evaluación

\begin{tabular}{ccc}
\hline Nombre de la prueba & Evaluación Pre & Evaluación Post \\
\hline Nombre de letras & Normal & Lento \\
Igual o diferente & Normal & Normal \\
Lectura de Palabras & Normal & Normal \\
Lectura de & Normal & Normal \\
$\begin{array}{c}\text { Pseudopalabras } \\
\text { Signos de } \\
\text { puntuación }\end{array}$ & Normal & Normal \\
\hline
\end{tabular}

Los resultados de todas las áreas se encontraban dentro de lo normal en los dos momentos, excepto la lectura del nombre de las letras, que pasa de normal a lento. 
La última prueba empleada fue la de Evaluación del lenguaje Oral al Lenguaje Escrito (Loleva) (Fernández Amado et al., 2006; Mayor et al., 2011). En la tabla 4 se muestran los resultados en conciencia fonológica.

Tabla 4

Resultados Loleva en conciencia fonológica en las dos fases de evaluación

\begin{tabular}{|c|c|c|c|}
\hline \multicolumn{2}{|c|}{ Evaluación pre } & \multicolumn{2}{|c|}{ Evaluación post } \\
\hline $\begin{array}{l}\text { Nombre de la } \\
\text { prueba }\end{array}$ & Pts & $\begin{array}{c}\text { Nombre de la } \\
\text { prueba }\end{array}$ & Pts \\
\hline Rima & $7 / 10$ & Rima & $7 / 10$ \\
\hline $\begin{array}{l}\text { Identificación de } \\
\text { sílaba inicial }\end{array}$ & $3 / 5$ & $\begin{array}{l}\text { Identificación de } \\
\text { sílaba inicial }\end{array}$ & $4 / 5$ \\
\hline $\begin{array}{l}\text { Identificación de } \\
\text { sílaba final }\end{array}$ & $3 / 5$ & $\begin{array}{l}\text { Identificación de } \\
\text { sílaba final }\end{array}$ & $4 / 5$ \\
\hline $\begin{array}{l}\text { Adición de sílaba } \\
\text { inicial }\end{array}$ & $2 / 5$ & $\begin{array}{l}\text { Adición de sílaba } \\
\text { inicial }\end{array}$ & $5 / 5$ \\
\hline $\begin{array}{l}\text { Adición de sílaba } \\
\text { final }\end{array}$ & $2 / 5$ & $\begin{array}{l}\text { Adición de sílaba } \\
\text { final }\end{array}$ & $4 / 5$ \\
\hline $\begin{array}{l}\text { Omisión de sílaba } \\
\text { inicial }\end{array}$ & $5 / 5$ & $\begin{array}{c}\text { Omisión de sílaba } \\
\text { inicial }\end{array}$ & $5 / 5$ \\
\hline $\begin{array}{l}\text { Omisión de sílaba } \\
\text { final }\end{array}$ & $4 / 5$ & $\begin{array}{l}\text { Omisión de sílaba } \\
\text { final }\end{array}$ & $4 / 5$ \\
\hline $\begin{array}{l}\text { Identificación de } \\
\text { fonema inicial }\end{array}$ & $3 / 5$ & $\begin{array}{l}\text { Identificación de } \\
\text { fonema inicial }\end{array}$ & $5 / 5$ \\
\hline $\begin{array}{l}\text { Identificación de } \\
\text { fonema final }\end{array}$ & $4 / 5$ & $\begin{array}{l}\text { Identificación de } \\
\text { fonema final }\end{array}$ & $5 / 5$ \\
\hline $\begin{array}{c}\text { Adición de } \\
\text { fonema inicial }\end{array}$ & $2 / 5$ & $\begin{array}{c}\text { Adición de } \\
\text { fonema inicial }\end{array}$ & $2 / 5$ \\
\hline $\begin{array}{l}\text { Adición de } \\
\text { fonema final }\end{array}$ & $4 / 5$ & $\begin{array}{l}\text { Adición de } \\
\text { fonema final }\end{array}$ & $4 / 5$ \\
\hline $\begin{array}{l}\text { Omisión de } \\
\text { fonema inicial }\end{array}$ & $3 / 5$ & $\begin{array}{c}\text { Omisión de } \\
\text { fonema inicial }\end{array}$ & $4 / 5$ \\
\hline $\begin{array}{l}\text { Omisión de } \\
\text { fonema final }\end{array}$ & $5 / 5$ & $\begin{array}{l}\text { Omisión de } \\
\text { fonema final }\end{array}$ & $5 / 5$ \\
\hline
\end{tabular}

Si se comparan los resultados del Loleva antes y después de la intervención se pueden observar mejoras en la realización de tareas de conciencia fonológica. En el caso de las tareas que fueron objeto de intervención hubo variabilidad de resultados. En las tareas de identificación se produjeron avances positivos tanto en el caso de fonema como de sílaba. En las subpruebas de adición, tan sólo se aprecian mejoras a nivel de sílaba.

En cuanto a las subpruebas de competencia lectora, los avances fueron menos importantes. Aún así, en la lectura de letras, tanto mayúsculas como minúsculas, hubo una leve mejora. Las demás puntuaciones se mantuvieron prácticamente invariables tras la intervención. Estos resultados aparecen reflejados en la tabla 5 .

Tabla 5

Resultados Loleva en competencia lectora inicial en las dos fases de evaluación

\begin{tabular}{cccc}
\hline $\begin{array}{c}\text { Evaluación previa a la } \\
\text { intervención }\end{array}$ & \multicolumn{2}{c}{$\begin{array}{c}\text { Evaluación posterior a la } \\
\text { intervención }\end{array}$} \\
\hline $\begin{array}{c}\text { Nombre de la } \\
\text { prueba }\end{array}$ & $P t s$ & $\begin{array}{c}\text { Nombre de la } \\
\text { prueba }\end{array}$ & Pts \\
$\begin{array}{c}\text { Lectura de letras } \\
\text { mayúsculas }\end{array}$ & $25 / 29$ & $\begin{array}{c}\text { Lectura de letras } \\
\text { mayúsculas }\end{array}$ & $26 / 29$ \\
$\begin{array}{c}\text { Lectura de letras } \\
\text { minúsculas }\end{array}$ & $25 / 29$ & $\begin{array}{c}\text { Lectura de letras } \\
\text { minúsculas }\end{array}$ & $27 / 29$ \\
\hline
\end{tabular}

\begin{tabular}{cccc}
\hline $\begin{array}{c}\text { Lectura de } \\
\text { palabras regulares }\end{array}$ & $10 / 10$ & $\begin{array}{c}\text { Lectura de } \\
\text { palabras regulares }\end{array}$ & $10 / 10$ \\
$\begin{array}{c}\text { Lectura de } \\
\text { palabras } \\
\text { complejas }\end{array}$ & $10 / 10$ & $\begin{array}{c}\text { Lectura de palabra } \\
\text { complejas }\end{array}$ & $9 / 10$ \\
$\begin{array}{c}\text { Lectura de } \\
\text { pseudopalabras } \\
\text { Separación de } \\
\text { palabras }\end{array}$ & $9 / 10$ & $\begin{array}{c}\text { Lectura de } \\
\text { pseudopalabras } \\
\text { Separación de } \\
\text { palabras }\end{array}$ & $3 / 5$ \\
\hline
\end{tabular}

El tiempo empleado en la realización de las tareas de competencias lectoras fue prácticamente el mismo en la evaluación previa y en la posterior a la intervención en casi todas las actividades. Se aprecia una mejoría notable en lectura de pseudopalabras y en separación de palabras. A pesar de esto, en la tarea de lectura de letras mayúsculas se empleó casi el doble de tiempo en terminar la actividad tras la intervención que antes de iniciarla. Estos datos se recogen en la tabla 6.

Tabla 6

Tiempo empleado en las tareas de competencia lectora inicial del Loleva

\begin{tabular}{|c|c|c|c|}
\hline \multicolumn{2}{|c|}{$\begin{array}{l}\text { Evaluación previa a la } \\
\text { intervención }\end{array}$} & \multicolumn{2}{|c|}{$\begin{array}{l}\text { Evaluación posterior a la } \\
\text { intervención }\end{array}$} \\
\hline $\begin{array}{l}\text { Nombre de la } \\
\text { prueba }\end{array}$ & $\begin{array}{l}\text { Tiempo } \\
\text { (") }\end{array}$ & $\begin{array}{l}\text { Nombre de la } \\
\text { prueba }\end{array}$ & $\begin{array}{l}\text { Tiempo } \\
\text { (") }\end{array}$ \\
\hline $\begin{array}{l}\text { Lectura de letras } \\
\text { mayúsculas }\end{array}$ & 86 & $\begin{array}{l}\text { Lectura de letras } \\
\text { mayúsculas }\end{array}$ & 147 \\
\hline $\begin{array}{l}\text { Lectura de letras } \\
\text { minúsculas }\end{array}$ & 81 & $\begin{array}{l}\text { Lectura de letras } \\
\text { minúsculas }\end{array}$ & 87 \\
\hline $\begin{array}{l}\text { Lectura de } \\
\text { palabras } \\
\text { regulares }\end{array}$ & 23 & $\begin{array}{l}\text { Lectura de } \\
\text { palabras } \\
\text { regulares }\end{array}$ & 25 \\
\hline $\begin{array}{l}\text { Lectura de } \\
\text { palabras } \\
\text { complejas }\end{array}$ & 32 & $\begin{array}{l}\text { Lectura de } \\
\text { palabra } \\
\text { complejas }\end{array}$ & 33 \\
\hline $\begin{array}{c}\text { Lectura de } \\
\text { pseudopalabras }\end{array}$ & 54 & $\begin{array}{c}\text { Lectura de } \\
\text { pseudopalabras }\end{array}$ & 42 \\
\hline $\begin{array}{l}\text { Separación de } \\
\text { palabras }\end{array}$ & 133 & $\begin{array}{l}\text { Separación de } \\
\text { palabras }\end{array}$ & 111 \\
\hline
\end{tabular}

\section{Discusión}

El principal objetivo del presente estudio fue la estimulación del reconocimiento y adición de sílaba y fonema, habilidades fonológicas que resultaron más deficitarias en la evaluación previa a la intervención.

En primer lugar cabe distinguir entre tareas de sílaba y de fonema. En el momento de evaluación inicial la conciencia fonémica estaba ligeramente más desarrollada que la silábica, cuando se esperaba lo contrario. Esto puede deberse a dos razones principales. En primer lugar, hay que tener en cuenta el curso en el que se encontraba el niño en el momento del estudio, $1^{\circ}$ de Educación Primaria, momento educativo en el que se le da mucha importancia al desarrollo lector. Está demostrado que la competencia lectora se relaciona directamente con la conciencia fonémica, pues así lo revelan estudios como el de Alcock, Ngorosho, Deus y Jukes (2010) o el de de Eslava y Cobos (2008). Por otro lado, también hay evidencias de que las dificultades en el 
habla repercuten negativamente en el desarrollo de la conciencia silábica.

Después de la intervención planteada, se mejoró el nivel de conciencia fonológica. Sin embargo, teniendo en cuenta que el nivel inicial de conciencia silábica era menor que el de conciencia fonémica, se puede afirmar que la evolución a nivel de sílaba fue mayor. Esto coincide con la hipótesis de Herrera y Defior (2005) y Bandini et al. (2013) de que aparecen antes las habilidades silábicas que las fonémicas, siendo el nivel silábico el más fácil y rápido de aprender por los niños.

$\mathrm{Si}$ en vez de tener en cuenta si se trata de una subprueba silábica o fonémica se presta atención a la posición de palabra, inicial o final, parece haber una tendencia a realizar mejor las tareas silábicas en posición inicial y las fonémicas en posición final. Como el nivel de varianza entre una posición y otra no es muy alto, sería recomendable estudiar con mayor profundidad este aspecto para determinar conclusiones más precisas.

El segundo punto de estudio fue la relación entre conciencia fonológica y lectura. Estudios anteriores como el de Ramos (2004) revelan una relación recíproca entre conciencia fonológica y lectura. Tras este estudio queda demostrado que la estimulación de la conciencia fonológica favorece las habilidades lectoras.

Por otra parte, tras haber intervenido en conciencia fonológica los resultados de todas las subpruebas de lectura se situaban en un nivel normal en todos los índices, a excepción de la de nombre de letras, en la que hubo un pequeño retroceso. $\mathrm{Si}$ se analizan cuantitativamente los resultados se comprende mejor por qué hubo un pequeño retroceso; antes de la intervención la puntuación obtenida por I. se situaba dentro de la normalidad en los índices principales y de velocidad, aunque con muy poca diferencia con respecto a puntuaciones inferiores a la media. Después de la intervención se agruparon los resultados en el apartado de dificultad leve, a pesar de que las puntuaciones en lectura del nombre de las letras eran similares a los de la evaluación inicial. Además, en la evaluación posterior a la intervención, el PROLEC-R se aplicó después de terminar la última subprueba del Loleva; si se tiene en cuenta que la subprueba de nombre de letras es el primer apartado de la segunda prueba aplicada pudo haber influido el factor cansancio, pues el niño no había tenido tiempo de descanso ni de adaptación a la nueva prueba.

Una de las habilidades de conciencia fonológica en las que se entrenó al niño fue en identificación de fonema. Esto lo hizo consciente de sus errores, motivo por el cual pensaba más sus respuestas en las pruebas de lectura de letras. Por esta razón los tiempos de lectura de letras aumentaron bastante en la segunda aplicación del Loleva y PROLEC-R.

Es importante hacer mención a una limitación muy importante en la realización de este estudio, la duración de la intervención. Tan sólo se llevaron a cabo 8 sesiones, de media hora cada una. Teniendo en cuenta que se trataba de un niño con dificultades de aprendizaje lo ideal hubiese sido disponer, como mínimo, de unas 12 sesiones de intervención, de modo que en cada una se trabajase tan sólo uno de los objetivos propuestos y para una sola posición de palabra. De disponer de este número de sesiones también contaríamos con más tiempo para repasar aquellas tareas que resultasen más complicadas para el niño, en este caso la adición de fonemas. Además, sería preferible que las sesiones tuviesen una duración mayor, de unos 45 minutos.

Con el fin de comprobar que los avances alcanzados se mantienen en el tiempo, sería recomendable llevar a cabo un seguimiento a las dos semanas y al mes de haber finalizado la intervención.

Finalmente indicar que sería conveniente prolongar la estimulación en conciencia fonológica por lo menos hasta que la adquisición de la lectoescritura se hubiese completado, a modo de apoyo.

\section{Bibliografía}

Alcock, K. J., Ngorosho, D., Deus, C., y Jukes, M. C. H. (2010). We don't hace language at our house: Disentangling the relationship between phonological awareness, chooling, and literacy. British Journal of Educational Psychology, 80(1), 55-76.

Bandini, H. H.., Santos, F. H., y de Souza, D.G. (2013). Levels of Phonological Awareness, Working Memory, and Lexical Knowledge in Elementary School Children. Paidéia (USP. Ribeirao Preto. Impresso), 23(56), 329-338.

Cuetos, F., Rodríguez, B., Ruano, E. y Arribas, D. (2007). PROLEC-R. Batería de Evaluación de los Procesos Lectores, revisada. Madrid: TEA Ediciones.

Defior, S., y Serrano, F. (2011). Procesos Fonológicos Explícitos e Implícitos, Lectura y Dislexia. Revista Neuropsicología, Neuropsiquiatría y Neurociencias, 11(1), 79-94.

de Eslava, L. M., y Cobos, J. E. (2008). Conciencia fonológica y aprendizaje lector. Acta Neurologica Colombiana, 24 (S2), S55-63.

Fernández Amado, Mayor, Zubiauz, Tuñas, y Peralbo, (2006) y Mayor, Zubiauz, Peralbo, Fernández Amado, Tuñas y Brenlla, (2011). Evaluación del Lenguaje Oral y Lenguaje Escrito (Loleva). Madrid: Cepe.

Gómez, L. A., Duarte. A. M., Merchán, V., Aguirre, D. C., y Pineda, D. A. (2007) Conciencia fonológica y comportamiento verbal en niños con dificultades de aprendizaje. Universitas Psychologica, 6 (3), 571-580.

Gómez-Betancur, L.A., Pineda, D.A., y Aguirre-Acevedo, D.C. (2005). Conciencia fonológica en niños con trastorno de la atención sin dificultades en el aprendizaje. Revista de neurología, 40 (10), 581-586.

Herrera, L., y Defior, S. (2005). Una Aproximación al Procesamiento Fonológico de los Niños Prelectores: Conciencia Fonológica, Memoria Verbal a Corto Plazo y Denominación. Psykhe (Santiago), 14 (2), 81-95.

Mayor, A., \& Zubiauz, B. (2011) LOLE. Del Lenguaje Oral al Lenguaje Escrito. Madrid: Cepe.

Pérez-Vallejo, M. (2010). Dificultades de aprendizaje. Revista digital, 35(1), 1-10.

Portellano Pérez, J. A., Mateos Mateos, R., Martínez-Arias, R. Granados García Tenorio, M. J. y Tapia Pavón, A. y (2006). Cuestionario de madurez neuropsicológica infantil: Cumanin ( $3^{\mathrm{a}}$ ed.) Madrid: TEA ediciones. 